\title{
Regional Short-term Micro-climate Air Temperature Prediction with CBPNN
}

\author{
Lvwen Huang ${ }^{1,2,}{ }^{*}$, Lianliang Chen ${ }^{1}$, Qin Wang ${ }^{1}$, Siwen $\mathrm{Yan}^{1}$, Xunbing Gao ${ }^{1}$, and Jiangjiang Luan ${ }^{1}$ \\ ${ }^{1}$ College of Information Engineering, Northwest A\&F University, Yangling, 712100, P. R. China \\ ${ }^{2}$ Key laboratory of Agricultural Internet of Things, Ministry of Agriculture, P. R. China
}

\begin{abstract}
This paper proposes a novel short-term air temperature prediction with three-layer Back Propagation Neural Network (BPNN) for the regional application of next 1-12 hours. With the continuous collection of eight real-time micro-climate parameters in the experimentation and demonstration stations in our university, the Multiple Stepwise Regression (MSR) is employed to screen the original historical data to find the parameter factors with greater contribution rate. On the basis of the Root Mean Square Error (RMSE) value evaluating the optimal fitting degree of the stepwise regression, the Levenberg-Marquardt (LM) and the Resilient Propagation (R-Prop) training algorithm are employed to construct a Combined BPNN (CBPNN) with two MSR inputs. Compared with the known micro-climate data sets, the Mean Absolute Error (MAE) is to evaluate the applicability of CBPNN prediction model. The experimentation shows that the MAE is within $4^{\circ} \mathrm{C}$ in the next 12 hours. This proposal will be deployed in stations in our university for extreme weather warnings, and could be applied to some regional short-term parameter prediction for the future agricultural production service.
\end{abstract}

\section{Introduction}

Crop damages caused by extreme temperatures can generally cause crop damage and yield reduction, and is a serious matter to agricultural producers [1]. The Shaanxi Province is the largest apple producing areas in China, and also is the largest apple planting concentrated region in the world, which the total yield per year accounting for $1 / 3$ in China and 1/7 in the world. Apple fruit is one of the most sensitive crops to meteorological conditions, especially at the flowering period and mature period, and the great fluctuating of air temperature can reduce the yield and quality to certain extent [2]. Basically the fruit production region suffers with different extents of spring flowering freezing and autumn mature frost damage every year [3]. The damage loss extent is different due to the different micro-climate environment [4]. It is necessary to construct a prediction model of micro-climate parameter to reduce the local damage loss of yield and quality.

The real-time micro-climate parameters collected at various experimental and demonstration stations in our University can be publicly accessed on the website and be downloaded for the any researchers of our University (sf.nwsuaf.edu.cn/index.html). All the micro-climate parameters of stations only are shown real-time data for users, and will be used for a stable and robust all-weather forecasting model with Artificial Neural Network (ANN). Here, this paper focuses on the accuracy of micro-climate prediction model to guide the production of agriculture.

For the earliest applications of short term meteorological element forecasting with ANNs, the researchers of USA at first employed a Feed-Forward Neural Network (FFNN) prediction models which was superior to the traditional regressions [5]. In case of potential losses due to extreme cold and heat of crops and livestock in most of the south-eastern USA, Smith et al. [4] explored the role of Ward-style ANNs based on near real-time data in annual temperature forecasting. The air and dew point temperature predictions in Georgia with web-based ANN were employed for the Automated Environment Monitoring Network (AEMN) data [6-7]. With the ground-breaking applications of ANNs, the architecture of the ANNs plays a key role in the prediction capabilities.

With the development and application of ANN models, except for USA, the other countries have developed the short term meteorological parameter warnings and predictions of relative models. Kaur et al. [8]developed five different ANN models to predict short-term wind speed for predicting power generation from wind turbines in Indian Electricity. Nogay et al. [9] developed a number of different ANN models with 60 neurons used in Mardin area in Turkey. Feng et al. [10] used a hybrid artificial neural network model combining air quality trajectory analysis and wavelet transform to study and predict the average concentration of PM2.5 in the next two days. Castaneda-Mirand and Castan [11] designed and implemented a multi-layer perceptron ANNs with the Levenberg-Marquardt (LM) backpropagation algorithm for greenhouse frost control in central Mexico. The researchers of China Research Institute of Water Resources and Hydropower [12] presented an ANN

\footnotetext{
* Corresponding author: huanglvwen@nwsuaf.edu.cn
} 
model based on feed-forward back-propagation and improved by the LM algorithm to forecast the ice conditions of the Yellow River in the Inner Mongolia region. Most related researches of the meteorological elements predictions are having a particular significance in different hydrology, climatology and agronomics.

Due to the practical requirements of apple fruit production industry, the micro-climate air temperature prediction with ANN models is presented as a regional production making-decision support, shown in Fig. 1. For the eight-element micro-climate data sets including air temperature, air humidity, soil temperature, soil moisture, total radiation, wind direction, wind speed, and rainfall factor, a Combined Back Propagation Neural Network (CBPNN) model using LM algorithm and Resilient propagation (R-Prop) training algorithm are designed. Compared with the traditional of Multiple Stepwise Regression (MSR) method, the key evaluation index of Mean Absolute Error (MAE) is employed to evaluate the fitness of prediction.

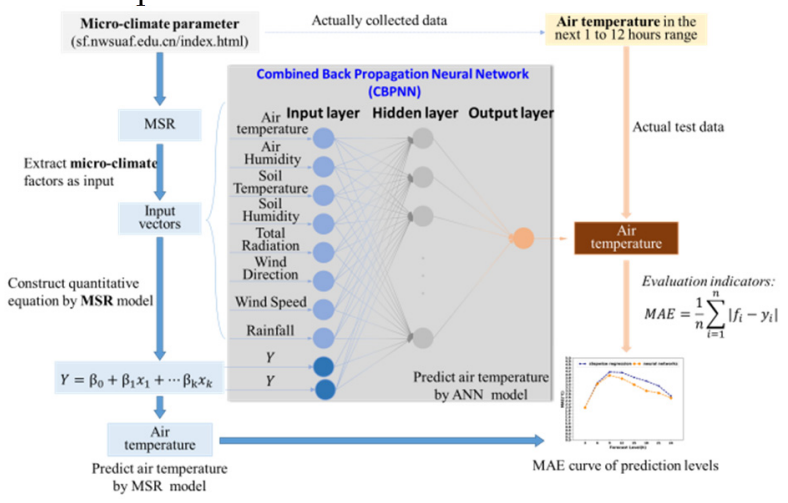

Fig.1. The flowchart of short-term and regioanl micro-climate air temperature prediction with CBPNN models.

At section two in this paper, the collection of data sets and prediction model design of MSR and BPNN are made. Section three carries out the MAE evaluation experimentation of MSR and CBPNN models. Section four concludes this paper.

\section{Methodologies}

\subsection{Data Sets}

The micro-climate parameters are automatically collected every one minute at the various stations in our University and the average value of every parameter are stored every 10 minutes. Due to the apple production technology and farming of Shaanxi Baishui Apple Experimentation and Demonstration Station (simplified as Baishui Station below) in our University could radiate and guide apple production in Loess Plateau which is the largest highquality apple fruit planting concentrated region in the world, the data set of Baishui Station is selected of totally over 60,000 records. The eight micro-climate factors includes air temperature, air humidity, soil temperature, soil moisture, total radiation, wind direction, wind speed and rainfall. The total micro-climate data is divided into training sets and test sets. The training sets contains the
49,605 records; $80 \%$ of the total data. The test sets contains the 12,000 records; $20 \%$ of the total data.

\subsection{Prediction Model of MSR}

To effectively and accurately construct the short-term air temperature prediction model, the significant factors are selected to find the correlation between the factor data [13]. For the eight possible input variates, it is necessary to screen the significant factors from the eight possible micro-climate factors. Whereas the optimal regression equation with the selected variables based, the MSR analytical model is expressed as equation (1) as follows,

$$
Y=\beta_{0}+\beta_{1} x_{1}+\cdots \beta_{\mathrm{k}} x_{k}
$$

where $Y$ is the observation dependent variable; $\beta_{0}$ presents the regression constant; $\beta_{\mathrm{k}}$ is the partial regression coefficient of the independent variable $x_{k} ; k$ is the number of independent variables, here $k$ is eight. The values of the partial regression coefficients $\beta_{1} \ldots \beta_{\mathrm{k}}$ and the regression constant $\beta_{0}$ in the equation (1) can be calculated by the data analysis of the training samples.

The Residual Standard Deviations Error (RMSE) is employed to evaluate the precision of the MSR model by comparing the deviation between the predicted and real data, and is calculated by equation (2) below:

$$
R M S E=\sqrt{\frac{1}{n} \sum_{i=1}^{n}\left(f_{i}-y_{i}\right)^{2}}
$$

where $n$ is the total number of the training data set, here it is 49,$605 ; f_{i}$ is the predicted value; $y_{i}$ is the actual observed value.

After the upper limit of significance probability is set as 0.05 , and the lower limit as 0.10 , the MSR model is trained by the sample data sets. Obtaining the significance test of the MSR equation (1), the partial regression coefficients, the regression constants, and RMSE of the eight micro-climate factors for the 12 stepwise regression models between 1 and 12 hours are obtained, shown in Table 1 below. From Table 1, with the increasing of the prediction hour level, the RMSE of MSR model shows the trend of continuously increasing, and reaches the maximum value 4.2650 at the prediction level of $9 \mathrm{~h}$, where shows the worst fitting effect. The effect lies at the minimum RMSE of 0.9493 , and stands at the initial prediction level of $1 \mathrm{~h}$.

\subsection{Prediction Model of CBPNNs}

From the Table 1, only the best prediction level of one hour could not meet the demands of actual practice because the MSR model is suitable for the linear relationship among the dependent variable and multiple variables. The BPNN with three-layer widely used has the ability to simulate any complex nonlinear mapping or function approximation [14]. Here according to Fig. 1, the input layer has eight variables of micro-climate elements, and the output layer one variable of air temperature. To reduce the impact of different dimensions of BPNN training and forecasting and to avoid the ANN unit 
saturation [12], the samples data is normalized to $[-1,1]$ as equation (3) as follows:

$$
y=\frac{\left(\mathrm{y}_{\max }-y_{\min }\right) *\left(x-x_{\min }\right)}{\mathrm{x}_{\max }-x_{\min }}+y_{\min }
$$

where $y$ is the normalized variable, and $y_{\max }$ and $y_{\min }$ are 1 and -1 , respectively; $x$ is the initial variable, and $x_{\max }$ and $x_{\min }$ are the maximum and minimum value of the variable $x$ respectively.

Table 1. The series of parameters of 12 MSR prediction model at level of 1-12 hours.

\begin{tabular}{|c|c|c|c|c|c|c|c|c|c|c|}
\hline \multirow[b]{2}{*}{$\begin{array}{l}\text { Prediction } \\
\text { Level } \\
\text { (hour) }\end{array}$} & \multicolumn{8}{|c|}{ Eight partial regression coefficient $\beta$ of the input micro-climate factors } & \multirow[b]{2}{*}{$\begin{array}{c}\text { Regression } \\
\text { Constant } \\
\beta_{0}\end{array}$} & \multirow[b]{2}{*}{ RMSE } \\
\hline & $\begin{array}{c}\text { Air } \\
\text { Temperature } \\
\left({ }^{\circ} \mathrm{C}\right)\end{array}$ & $\begin{array}{c}\text { Air } \\
\text { Humidity } \\
(\% \mathrm{RH})\end{array}$ & $\begin{array}{c}\text { Soil } \\
\text { Temperature } \\
\left({ }^{\circ} \mathrm{C}\right)\end{array}$ & $\begin{array}{l}\text { Soil } \\
\text { Humidity } \\
(\% \mathrm{VWC})\end{array}$ & $\begin{array}{c}\text { Total } \\
\text { Radiation } \\
(\mathrm{W} / \mathrm{m} 2)\end{array}$ & $\begin{array}{c}\text { Wind } \\
\text { Direction } \\
\left(^{\circ}\right)\end{array}$ & $\begin{array}{l}\text { Wind } \\
\text { Speed } \\
(\mathrm{m} / \mathrm{s})\end{array}$ & $\begin{array}{c}\text { Rainfall } \\
(\mathrm{mm})\end{array}$ & & \\
\hline 1 & 0.9008 & 0.0134 & 0.0868 & -0.9798 & 0.0034 & -0.0005 & 0.0830 & -0.2565 & -0.9080 & 0.9493 \\
\hline 2 & 0.7818 & 0.0256 & 0.2019 & -2.2086 & 0.0060 & -0.0011 & 0.1809 & -0.4017 & -1.6547 & 1.6491 \\
\hline 3 & 0.6542 & 0.0358 & 0.3323 & -3.5540 & 0.0077 & -0.0019 & 0.2780 & -0.5216 & -2.1620 & 2.2990 \\
\hline 4 & 0.5269 & 0.0436 & 0.4673 & -4.9389 & 0.0085 & -0.0027 & 0.3596 & -0.6269 & -2.4129 & 2.8794 \\
\hline 5 & 0.4084 & 0.0492 & 0.5980 & -6.2954 & 0.0084 & -0.0035 & 0.4255 & -0.8065 & -2.4402 & 3.3698 \\
\hline 6 & 0.3047 & 0.0527 & 0.7166 & -7.5431 & 0.0076 & -0.0043 & 0.4746 & -1.0215 & -2.2699 & 3.7542 \\
\hline 7 & 0.2199 & 0.0541 & 0.8178 & -8.6337 & 0.0062 & -0.0049 & 0.5007 & -1.2138 & -1.9431 & 4.0282 \\
\hline 8 & 0.1568 & 0.0540 & 0.8962 & -9.5336 & 0.0044 & -0.0054 & 0.5032 & -1.3659 & -1.4814 & 4.1938 \\
\hline 9 & 0.1166 & 0.0527 & 0.9493 & -10.2251 & 0.0024 & -0.0059 & 0.4828 & -1.4334 & -0.9288 & 4.2650 \\
\hline 10 & 0.1005 & 0.0507 & 0.9728 & -10.6812 & 0.0004 & -0.0064 & 0.4380 & -1.4829 & -0.2762 & 4.2625 \\
\hline 11 & 0.1090 & 0.0476 & 0.9669 & -10.8771 & -0.0017 & -0.0069 & 0.3637 & -1.5858 & 0.4590 & 4.2114 \\
\hline 12 & 0.1429 & 0.0434 & 0.9304 & -10.7556 & -0.0038 & -0.0075 & 0.2706 & -1.6568 & 1.2513 & 4.1280 \\
\hline
\end{tabular}

The LM and the R-Prop are employed to design the BPNN prediction model, where the LM has the fastest convergence performance to the non-constrained multidimensional nonlinearity [15] and the R-Prop can effectively eliminate the partial derivative size [16]. The activation function between the input layer and the hidden layer is set as the hyperbolic tangent function of Tansig, and the activation function between the hidden layer and the output layer as the linear function of Purelin. With the BPNN trained with sample data sets, the prediction models between 1-12 hours are obtained. The MAE is used to measure the specific model accuracy [1], expressed in equation (4) expressed below,

$$
M A E=\frac{1}{n} \sum_{i=1}^{n}\left|f_{i}-y_{i}\right|
$$

where $f_{i}$ is the prediction value; $y_{i}$ is the actual observation value, and here it represents the test sample data; and $n$ is the total number of the data set.

\section{Experimentations and Discussions}

After the BPNN trained by the sample data, twelve prediction models at level of 1-12 hours are obtained. The twelve BPNN prediction models are tested with sample data of 12,000 records, and the MAEs of equation (4) are calculated to evaluate the precision of models. Compared with MSR prediction models, shown in Table 2, the BPNN prediction models only have the excellence at the prediction level of 4-6h and 9-12h, and both the prediction methods could have a little higher errors. The traditional BPNN is generally used for fitting the data, and should be optimized for this prediction. To achieve better prediction accuracy and to reduce the time cost, the constant adjusting of the number of hidden layers and nodes, the activation and training functions of the network should be taken to find out the optimal learning network.

Table 2. The MAEs comparisons of MSR and BPNN prediction models.

\begin{tabular}{c|c|c|c|c|c|c|c|c|c|c|c|c}
\hline \multirow{2}{*}{ Prediction Model } & \multicolumn{10}{c}{ The MAEs at prediction Level(h) } \\
\cline { 2 - 27 } & 1 & 2 & 3 & 4 & 5 & 6 & 7 & 8 & 9 & 10 & 11 & 12 \\
\hline MSR & 0.7789 & 1.3757 & 1.9758 & 2.5295 & 3.0443 & 3.4742 & 3.8020 & 4.0228 & 4.1426 & 4.1842 & 4.1661 & 4.1127 \\
\hline BPNN & 0.7698 & 1.3599 & 1.9758 & $\mathbf{2 . 4 7 5 4}$ & $\mathbf{2 . 9 4 8 8}$ & $\mathbf{3 . 4 1 5 7}$ & $\mathbf{3 . 6 9 1 1}$ & 3.8211 & $\mathbf{3 . 9 4 4 9}$ & $\mathbf{3 . 9 9 6 0}$ & $\mathbf{3 . 9 4 9 8}$ & $\mathbf{3 . 7 4 8 3}$ \\
\hline
\end{tabular}

A newly Combined BPNN (CBPNN) model is proposed. According to the neural network structure of Fig. 1, the predictive value $Y$ obtained by the MSR prediction model is used as a new input to this learning network. To increase the weight of the predicted value $Y$ in this learning network, the input layer of CBPNN model changes into ten input variables. The input vector $\mathbf{D} \in R^{10}$, $\mathbf{D}=\left(Y, Y, X_{1}, X_{2}, X_{3}, X_{4}, X_{5}, X_{6}, X_{7}, X_{8}\right)^{T}$ represents in turn ten variables of prediction value (MSR prediction output $Y$ ), prediction value (MSR prediction output $Y$ ), air 
temperature, air humidity, soil temperature, soil moisture, total radiation, wind direction, wind speed and rainfall. The combined neural network model is employed at eight prediction levels of 4-7h and 9-12h, which is the bold and filled grey in Table 2 and has a little higher errors. The activation functions employed at prediction levels of $1 \mathrm{~h}$ and $2 \mathrm{~h}$ are Tansig function and Purelin function and the other ten prediction levels are Tansig function. The training functions employed at prediction levels of 1-3h become LM algorithm and at the other ten prediction levels are R-Prop algorithm. The experimentation verifies that this CBPNN model prediction models have higher

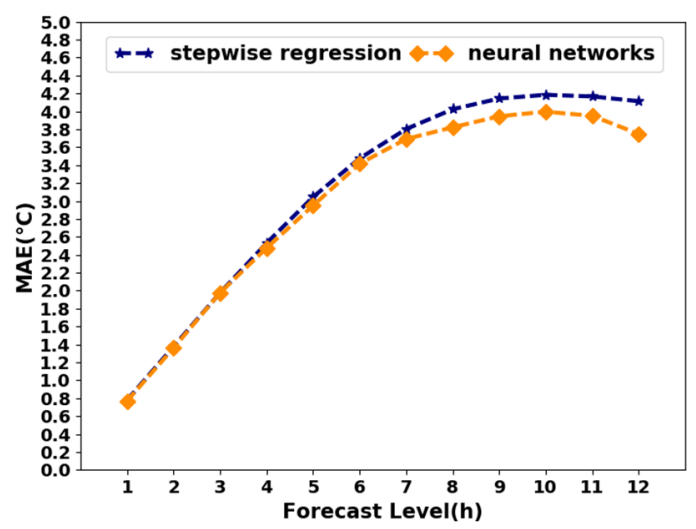

Fig. 2. The MAE comparison curves at predicted 12 hours. accuracy than MSR models at levels of 1-12 hours, shown in Fig. 2.

From the MAEs curves of Fig. 2, the errors of CBPNN prediction models are mostly smaller than it of MSR models. The average of MAEs of 12 prediction levels obtained by the MSR is $3.1341^{\circ} \mathrm{C}$ while the average of MAEs of 12 prediction levels obtained by the CBPNN model is $3.0081^{\circ} \mathrm{C}$, where the CBPNN model can effectively improve the prediction accuracy of air temperature with an average reduction of $0.1260^{\circ} \mathrm{C}$. At the prediction level of $10 \mathrm{~h}$, the MAEs reaches the highest, and the MAE of MSR model $4.1842^{\circ} \mathrm{C}$ and the MAE of the CBPNN model $3.9960^{\circ} \mathrm{C}$.

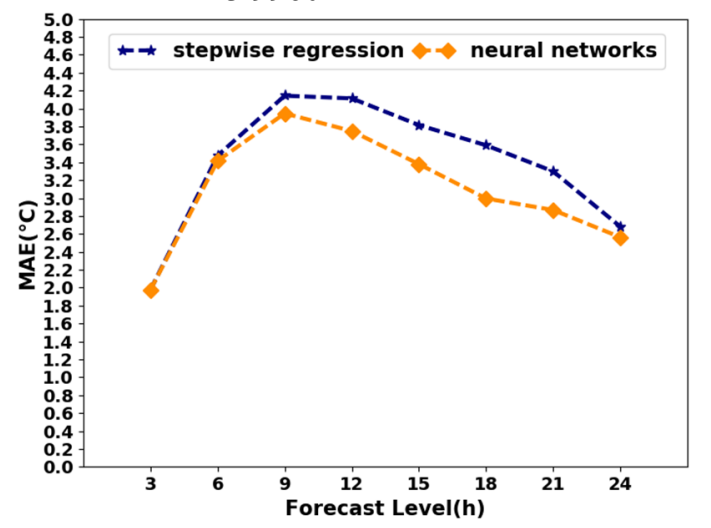

Fig. 4. The MAE comparison curves of prediction level of 24 hours between MSR and CBPNN models.

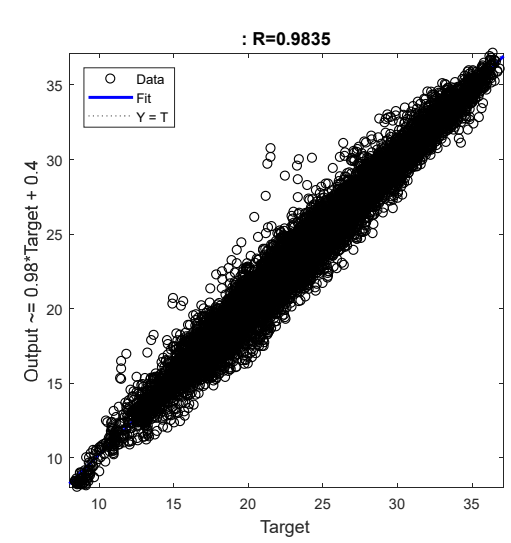

(a) Predict level at one hour

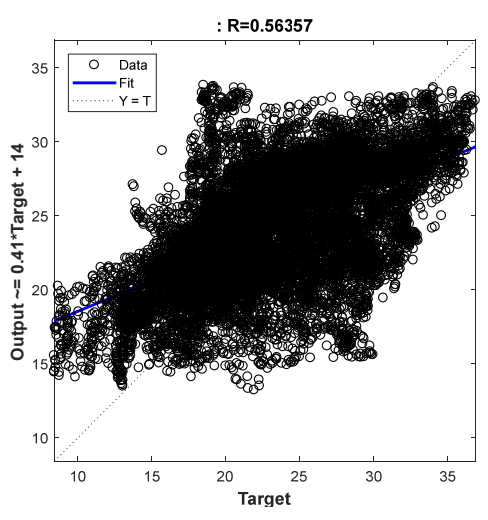

(b) Predict level at ten hours

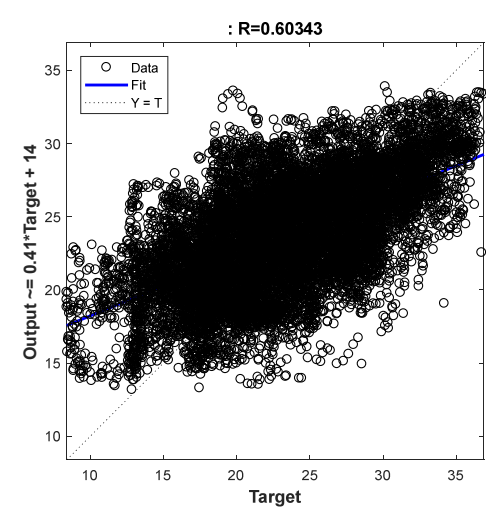

(c) Predict level at twelve hours

Fig. 3. Regression coefficient $R$ values at $1 \mathrm{~h}, 10 \mathrm{~h}$ and $12 \mathrm{~h}$ predicted levels.

The neural network model is used to achieve the optimal prediction performance by changing the number of hidden layers and nodes and the training functions. With the prediction level increases, the training function of R-Prop algorithm is superior to it of the LM training, shown in Fig. 3. Actually, the resilient $R$ value shows the trend of decreasing as the prediction level increases. From the scatter plot of air temperature predictions in Fig. 3, the resilient $R$ value of the CBPNN model at the prediction level of $10 \mathrm{~h}$ reaches the lowest value of 0.56357 , while the slope of the regression line of MSR model is 0.41 and the intercept of 14 .

Generally as the prediction level increases, the MAE of the prediction model is increasing while the prediction accuracy is reducing. However, actually the MAE of prediction level 11-12h shows a downward trend. With the experimentation of 24 hours prediction, Table 3 below shows the MAE of the next 24 hours prediction levels of MSR and CBPNN models, which reflects the parabolic downward trend with the prediction level increases, and its corresponding curve shown in Fig. 4.

From the Table 3 and Fig. 4, if the prediction range within 24 hours, the average error of air temperature at level of $6-15 \mathrm{~h}$ is over $3^{\circ} \mathrm{C}$, while the average error at the other level is less than $3^{\circ} \mathrm{C}$.

\section{Conclusions}

This paper proposes a new CBPNN model to predict a regional and short-term air temperature with a level of 112 hour. The contribution lies at the MSR output as a new 
input to the BPNN, which can improve the prediction accuracy. The MAEs of CBPNN prediction models could be parabolic-like declining with the prediction level of hour's increasing.

Table 3. The MAEs comparisons of MSR and CBPNN prediction models at 24-hour predictive level.

\begin{tabular}{c|c|c|c|c|c|c|c|c}
\hline \multirow{2}{*}{ Prediction Mode } & \multicolumn{7}{c}{ The MAEs at prediction Level(h) } \\
\cline { 2 - 10 } & 3 & 6 & 9 & 12 & 15 & 18 & 21 & 24 \\
\hline MSR & 1.9758 & 3.4742 & 4.1426 & 4.1127 & 3.8150 & 3.5892 & 3.2982 & 2.6816 \\
\hline CBPNN & 1.9758 & $\mathbf{3 . 4 1 5 7}$ & $\mathbf{3 . 9 4 4 9}$ & $\mathbf{3 . 7 4 8 3}$ & $\mathbf{3 . 3 7 9 0}$ & $\mathbf{2 . 9 9 5 3}$ & $\mathbf{2 . 8 6 6 9}$ & $\mathbf{2 . 5 6 3 6}$ \\
\hline
\end{tabular}

With the experimentation of data sets collected at Baishui Station in our University, the CBPNN prediction model has more precisions and better convergence compared with BPNN or MSR model. This proposal will be deployed for micro-climate warning of extreme parameters and be extended for a regional short-term element forecasting decision to guide agriculture production. With this proposal, in the future we will explore the other parameter screening, data fittings, and growth parameter measurements.

\section{Acknowledgements}

This research was fully supported by National College Students Innovation and Entrepreneurship Training Program (No. 201610712063) and partially supported by Major Pilot Projects of the Agro-Tech Extension and Service in Shaanxi (No. 2016XXPT-00).

\section{References}

1. R.F. Chevalier, G. Hoogenboom, R.W. Mcclendon, J.A. Paz. Support vector regression with reduced training sets for air temperature prediction: a comparison with artificial neural networks. NEURAL COMPUT APPL, 20(1):151-159 (2011).

2. M. Pramsohler, J. Hacker, G. Neuner. Freezing pattern and frost killing temperature of apple (Malus domestica) wood under controlled conditions and in nature. TREE PHYSIOL, 32(7):819-828 (2012).

3. C.H. Greenberg, D.J. Levey, D.L. Loftis. Fruit Production in Mature and Recently Regenerated Forests of the Appalachians. J WILDLIFE MANAGE, 71(2):321-335 (2011).

4. S. Briana, H. Gerrit, M.C. Ronaldw. Artificial neural networks for automated year-round temperature prediction. COMPUT ELECTRON AGR, 68(1):52-61 (2009).

5. J. Yi, V.R. Prybutok. A neural network model forecasting for prediction of daily maximum ozone concentration in an industrialized urban area. ENVIRON POLLUT, 92(3):349-357 (1996).

6. K. Nadig, W. Potter, G. Hoogenboom, R. Mcclendon. Comparison of individual and combined ANN models for prediction of air and dew point temperature. APPL INTELL, 39(2):354-366 (2013).

7. D.B. Shank, G. Hoogenboom, R.W. Mcclendon. Dewpoint Temperature Prediction Using Artificial Neural Networks. J APPL METEOROL CLIM, 47(6):1757-1769 (2006).
8. T. Kaur, S. Kumar, R. Segal. Application of artificial neural network for short term wind speed forecasting. In: 2016 - Biennial International Conference on Power and Energy Systems: Towards Sustainable Energy, January 21-23, 2016, Bengaluru, India: 1-5 (2016).

9. H.S. Nogay, T.C. Akinci, M. Eidukeviciute. Application of artificial neural networks for short term wind speed forecasting in Mardin, Turkey. $J$ ENERGY SOUTH AFR, 23(4):2-7 (2012).

10. X. Feng, Q. Li, Y. Zhu, J. Hou, L. Jin, J. Wang. Artificial neural networks forecasting of PM2.5 pollution using air mass trajectory based geographic model and wavelet transformation. ATMOS ENVIRON, 107:118-128 (2015).

11. A. Castaeda-Miranda, V.M. Castao. Smart frost control in greenhouses by neural networks models. COMPUT ELECTRON AGR, 137:102-114 (2017).

12. T. Wang, K. Yang, Y. Guo. Application of Artificial Neural Networks to Forecasting Ice Conditions of the Yellow River in the Inner Mongolia Reach. $J$ HYDROL ENG, 13(9):811-816 (2008).

13. S. Wiesner, A. Eschenbach, F. Ament. Urban air temperature anomalies and their relation to soil moisture observed in the city of Hamburg. METEOROL Z, 23(2):143-157(2014)

14. K.W. Chau. Reliability and performance-based design by artificial neural network. $A D V E N G$ SOFTW, 38(3):145-149 (2007).

15. A.J. Adeloye, A.D. Munari. Artificial neural network based generalized storage - yield - reliability models using the Levenberg - Marquardt algorithm. $J$ HYDROL, 326(1-4):215-230 (2006).

16. C. Igel, M. Hüsken. Empirical evaluation of the improved Rprop learning algorithms. Neurocomputing, 50(1):105-123 (2003). 